\title{
Deficiency in E3 Ubiquitin Ligase Parkin Exacerbates Chronic Alcohol Intake-Induced Cardiomyopathy through an Ambra1-Dependent Mechanism
}

\author{
Mingjie Yang ${ }^{1}$, Shuyi Wang ${ }^{2}$, Ne Wu ${ }^{1}$, Xihui $\mathrm{Xu}^{2}$, Yingmei Zhang ${ }^{1}$, and Jun Ren ${ }^{3}$ \\ ${ }^{1}$ Zhongshan Hospital Fudan University \\ ${ }^{2}$ University of Wyoming \\ ${ }^{3}$ University of Wyoming College of Health Sciences
}

May 5, 2020

\begin{abstract}
Background and purpose: Chronic alcohol intake provoked unfavorable geometric and functional changes in the heart along with altered autophagy. Parkin, a cytosolic E3 ubiquitin ligase encoded by PARK2 gene, governs mitochondrial homeostasis and mitophagy although its role in alcoholic cardiomyopathy remains unclear. Experimental approach: This study was designed to examine the role of Parkin in alcohol-induced cardiomyopathy. Adult male wild-type (WT) and PARKIN2 knockout (Parkin-/-) mice were placed on alcohol $(4 \%)$ or control diet for 8 weeks. Echocardiographic and cardiomyocyte mechanical properties, myocardial and mitochondrial morphology, autophagy and mitophagy were evaluated. GFP-LC3 puncta was employed to assess autophagosome formation. Key results: Our results revealed that alcohol intake led to unfavorable geometric and contractile changes (enlarged left ventricular chamber; decreased fractional shortening, ejection fraction, peak shortening and velocity of shortening/relengthening, prolonged relengthening duration), enlarged cardiomyocyte size and interstitial fibrosis, as well as mitochondrial swelling with cristae disarrangement and mitochondrial depolarization, the effects of which were exacerbated by Parkin deficiency. Alcohol consumption promoted autophagy and PINK1-Parkin-mediated mitophagy, the effects of which were cancelled off by Parkin knockout. Co-immunoprecipitation noted a tight interaction between Parkin and Ambra1 (autophagy and beclin1 regulator 1). In vitro study using neonatal rat cardiomyocytes revealed that Parkin transfection ameliorated ethanol-induced changes in autophagy. However, Ambra1 silencing negated Parkin-induced protection against ethanol-induced autophagy. Conclusions and implications: Taken together, these data suggest an integral role for Parkin in the face of alcoholic challenge possibly through its interaction with Ambra1 to promote autophagy and maintain mitochondrial homeostasis.
\end{abstract}

\section{Hosted file}

BJP_Parkin EtOH_021820.doc available at https://authorea.com/users/299279/articles/428732deficiency-in-e3-ubiquitin-ligase-parkin-exacerbates-chronic-alcohol-intake-inducedcardiomyopathy-through-an-ambra1-dependent-mechanism 

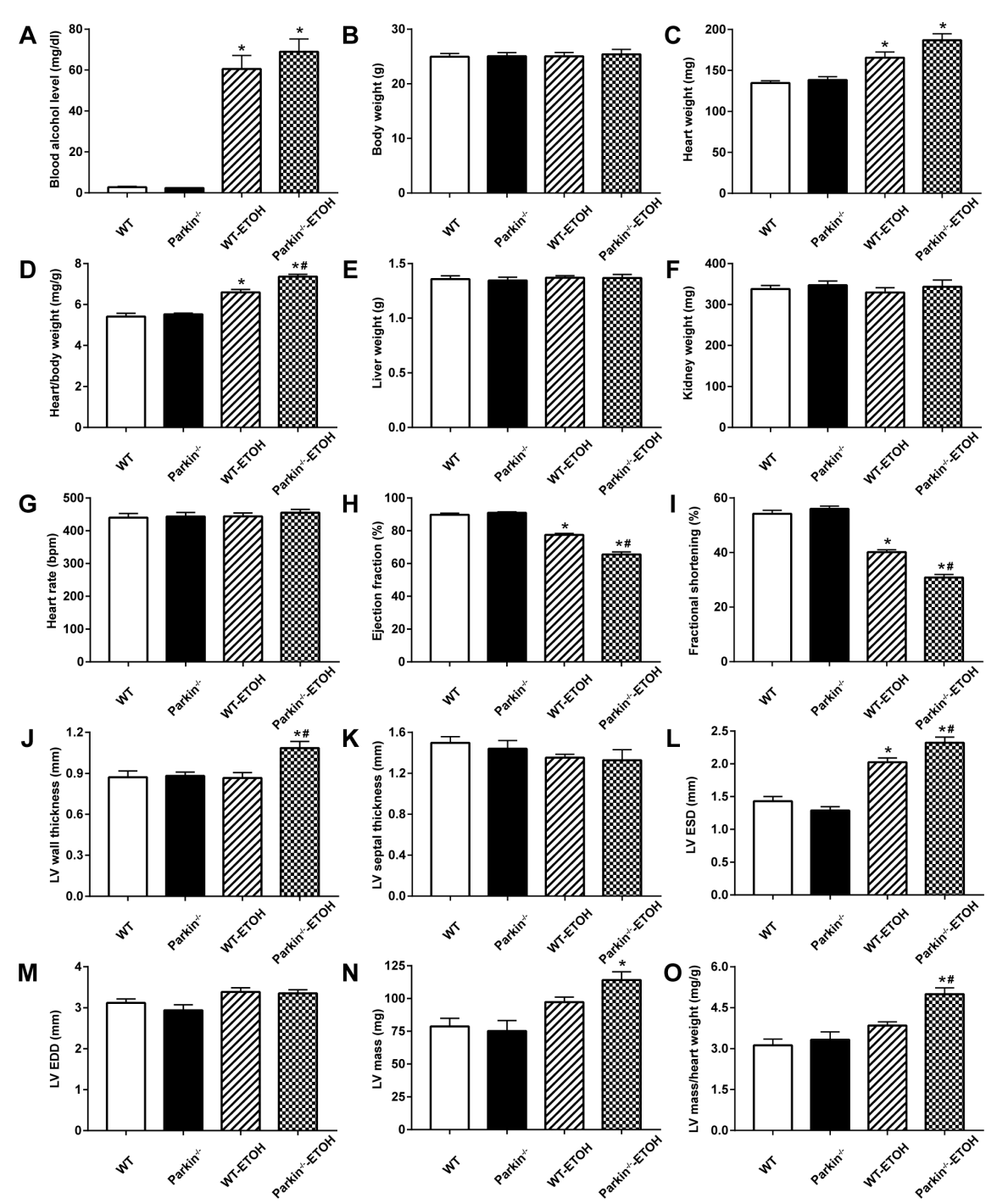

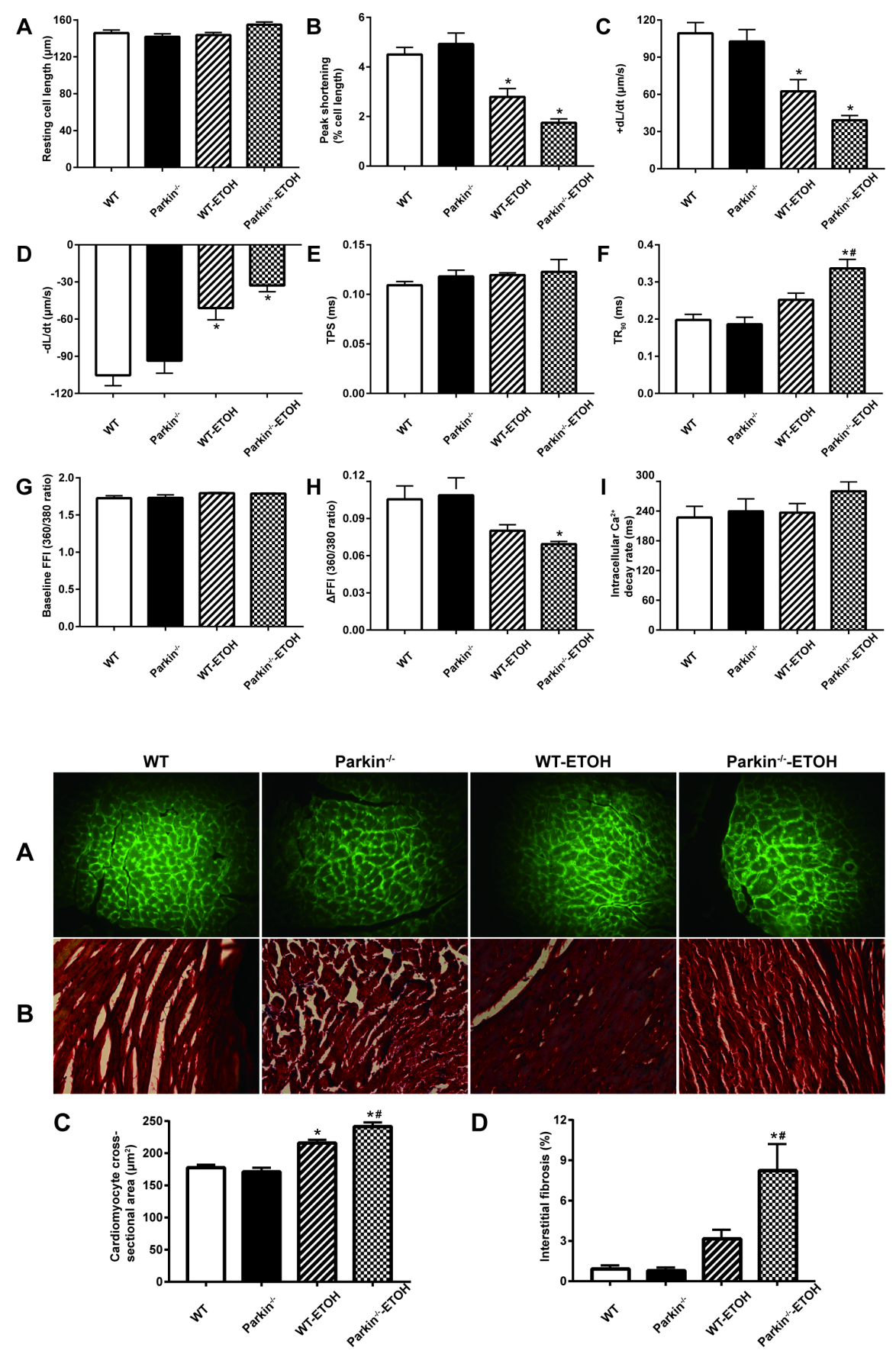


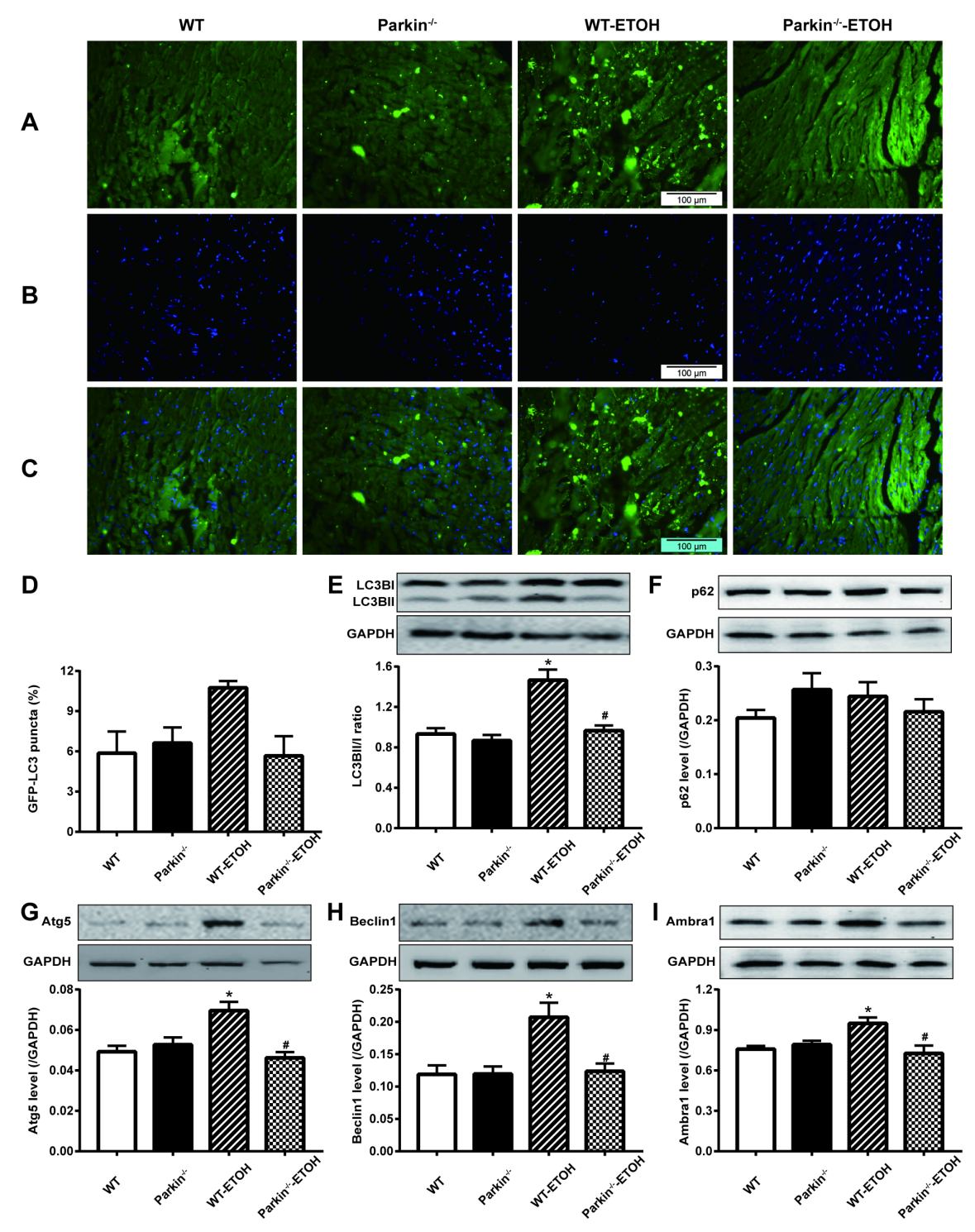


A
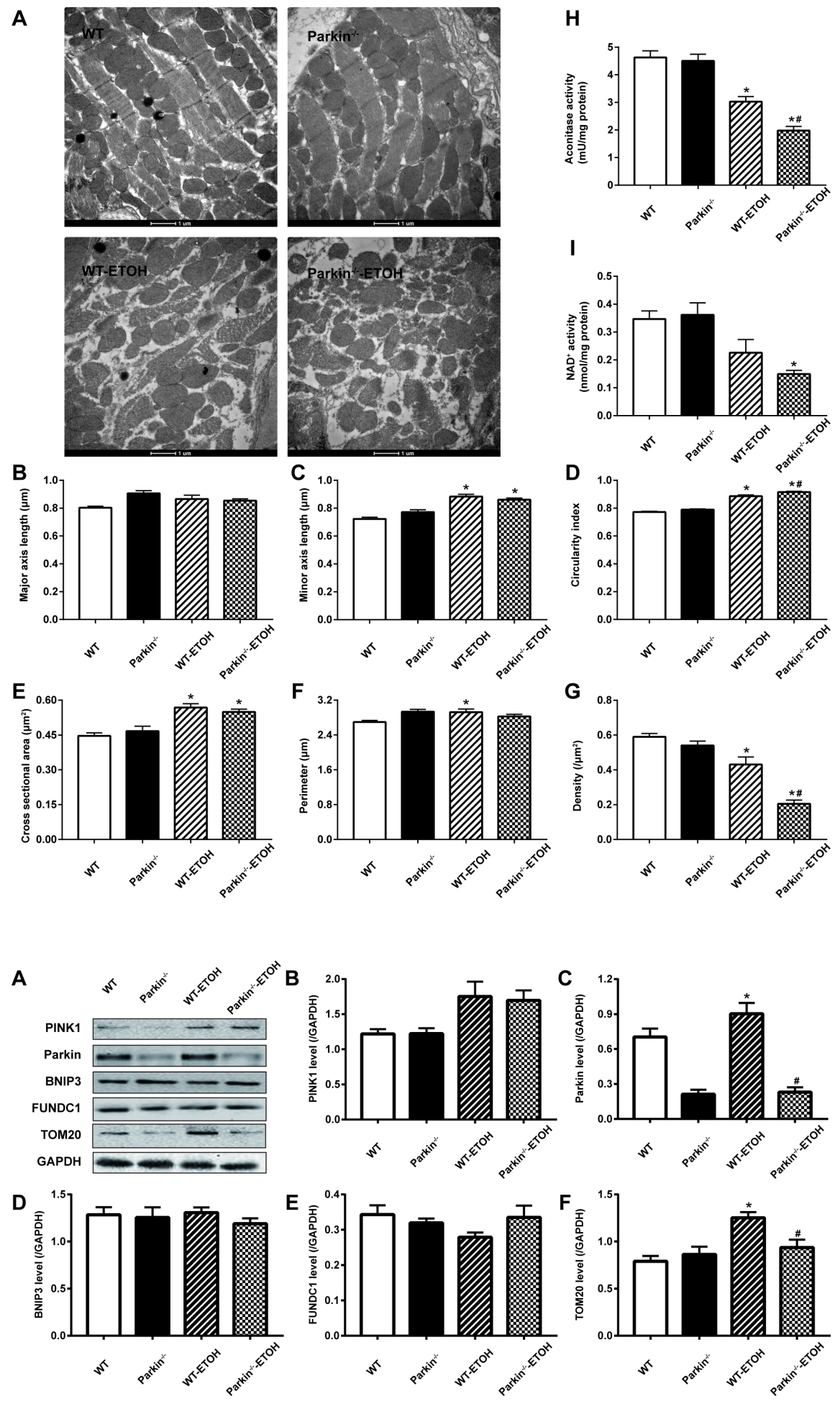
A
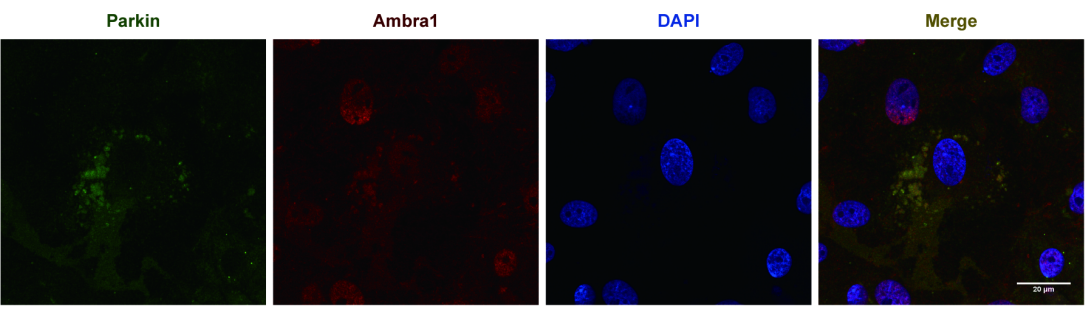

C

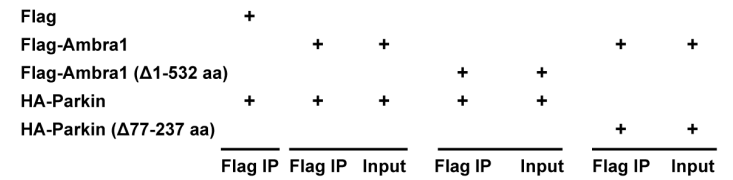

B

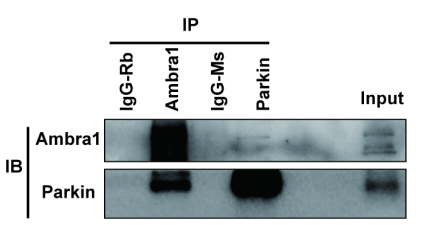

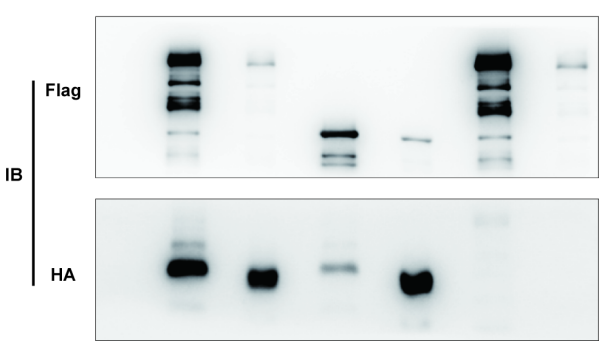

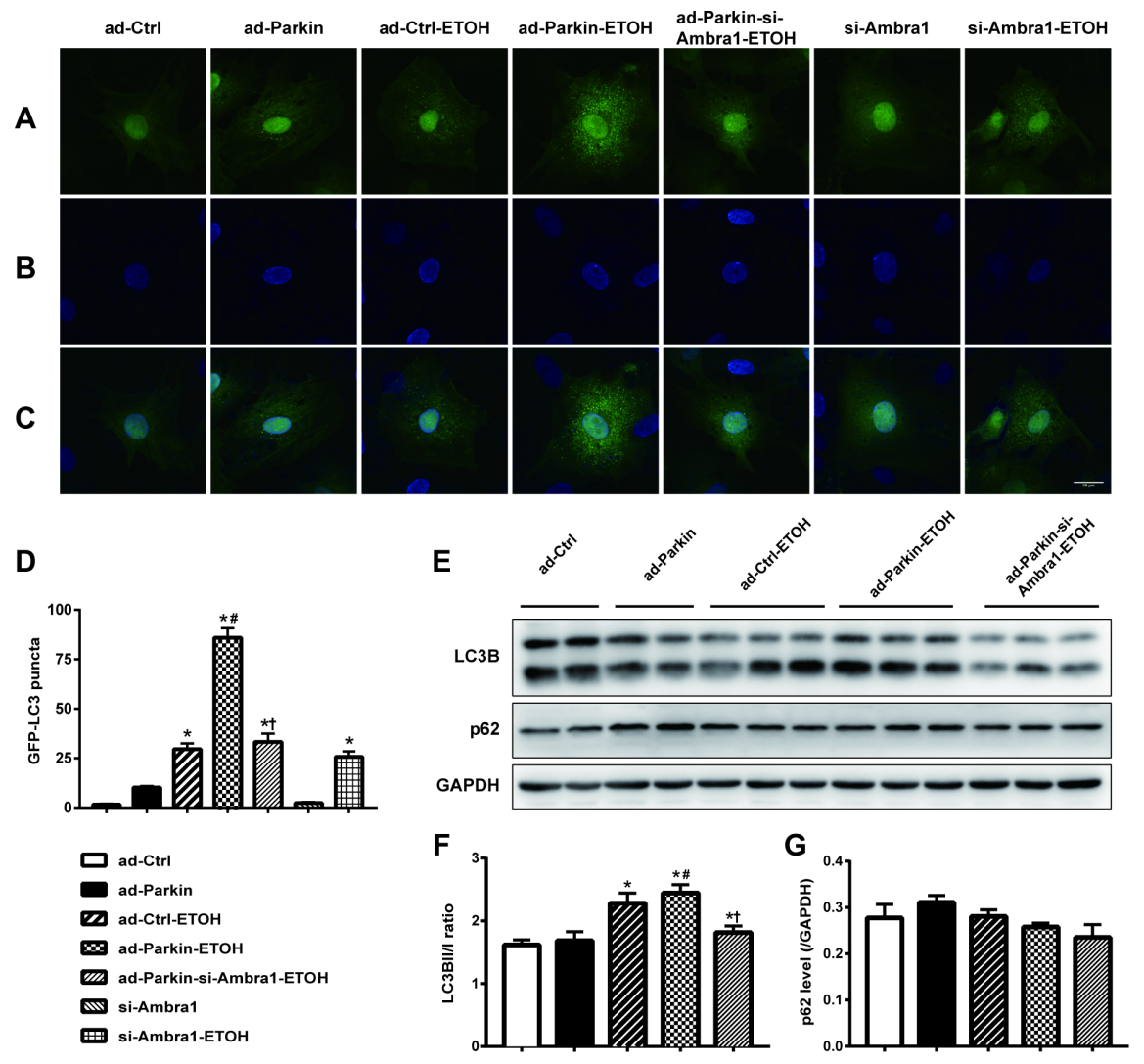

